\title{
Reagierende Schriftzeichen
}

\author{
Angelo Stitz ${ }^{1}$, Matthias Wölfel ${ }^{1,2}$ \\ Fakultät für Gestaltung, Hochschule Pforzheim ${ }^{1}$ \\ Fakultät für Digitale Medien, Hochschule Furtwangen²
}

\section{Zusammenfassung}

Erstmals in der Geschichte der Menschheit befähigt der Monitor die Ausgabe von variablen Inhalten. Die Entwicklung der Schrift und ihrer Zeichenformen entstammt jedoch aus dem statischen, analogen Medium. Digitale Ausgabegeräte in Verbindung mit Sensorik rücken das Individuum in den Focus und eröffnen die Möglichkeit, das Schriftzeichen nicht wie üblich als unveränderbar zu begreifen. Unser Ansatz eines „reagierenden Schriftzeichens“ folgt der Intention, sich in Echtzeit in seiner Zeichenform an die Begebenheiten des Lesers (z.B. Alter, Fehlsichtigkeit) und des Kontextes (z.B. Perspektive) anzupassen, um die Lesbarkeit der Schriftzeichen zu verbessern. Durch eine erste Umfrage konnten wir belegen, dass der Einsatz eines reagierenden Schriftzeichens sowohl die Lesbarkeit verbessert als auch auf hohe Akzeptanz trifft.

\section{Einführung}

Schrift fixiert Information, die sich - bis heute - in ihrer Zeichenform nicht verändert. Doch gibt es alternative Möglichkeiten? Erstmals in der Geschichte der Menschheit befähigt der Bildschirm die Ausgabe von variablen Inhalten. Die Entwicklung der Schrift und ihrer Zeichenform entstammt jedoch aus dem statischen, analogen Medium. Mit der Erfindung des Bleisatzes und damit der maschinellen Reproduktion von Schriftzeichen weitete sich der $\mathrm{Zu}-$ gang zu Wissen aus. Standardisierung ermöglichte Schnelligkeit in der Vermittlung von Information (Kinross 2010). Durch die industrielle Reproduktion von Schrift wurde die Zeichenform so optimiert, dass diese den Bedürfnissen eines durchschnittlichen Lesers entspricht. Schrift als substantielles Werkzeug der Informationsvermittlung findet sich jedoch zunehmend in einer kohärenten, vielseitigen Umgebung wieder. Die Umstände, in denen wir Information wahrnehmen und rezipieren, variieren sekündlich. Auch hat jedes Individuum eigene Bedürfnisse an die Zeichen, die von der Leseerfahrung, Sehschärft aber auch den Umgebungsbedingungen etc. abhängen.

Die Flexibilität des digitalen Mediums folgt keiner Limitierung eines analogen Mediums und kann in Verbindung mit Sensorik Bezug auf die Situationen und den Leser nehmen. Der Grundgedanke, eine visuelle Gestaltung auf variable Ausgabeformate (Smartphone, Desktop 
Monitor) anzupassen, stammte im Jahr 2010 von Ethan Marcottes und wurde unter dem Begriff Responsive Web Design bekannt. Wird dieser Ansatz auf die Zeichenform übertragen, eröffnet sich die Möglichkeit, das Schriftzeichen nicht wie üblich als unveränderbar zu begreifen. Bereits im Jahr 2013 stellte Marko Dugonji ein System vor, bei dem die Schriftgröße sich automatisch dem Leseabstand anpasst. Unser Ansatz eines „reagierenden Schriftzeichens“ (engl. ,responsive type“) geht über eine simple Größenveränderung hinaus. Er folgt der Intention, sich in seiner Zeichenform an die individuellen Eigenschaften des Lesers (z.B. Alter, Fehlsichtigkeit) und des Kontextes (z.B. Perspektive) anzupassen, um die Lesbarkeit der jeweiligen Zeichen zu verbessern. Diese Veränderung geht weit über eine einfache Größenveränderung hinaus und greift unmittelbar in die Form jedes einzelnen Zeichens ein.

\section{Reagierende Schriftzeichen}

Typografische Zeichen bezeichnen visuelle Symbole, zu denen u.a. Buchstaben, Ziffern, Interpunktion gehören. Die Zeichenform konstituiert sich durch die schwarze bedruckte Fläche (Smijers 2011), im Gegensatz zum umschließenden, unbedruckten Weißraum. Jedes Zeichen weist bestimmte Eigenschaften auf (Klanten et al. 2004), die in ihrer Ausprägung maßgeblich die Lesbarkeit eines Zeichens beeinflussen. Lesbarkeit beschränkt sich in diesem Kurzbeitrag auf die Erkennbarkeit und damit auf die Prägnanz eines einzelnen Zeichens. Diese nimmt auch wiederum Auswirkung auf die Erkennbarkeit eines Wortes, die sich hauptsächlich aus seinem Wortumriss ergibt, dem sog. Wortbild wie in Abbildung $1 \mathrm{zu}$ sehen. Im Gegensatz zu einer statischen Zeichenform kann ein reagierendes Schriftzeichen Bezug zu seinem Leser nehmen. Im Folgenden wollen wir drei Möglichkeiten skizzieren.

\subsection{Leseerfahrung}

Im Gegensatz zu unerfahrenen Lesern, die noch kein Wortbild verinnerlicht haben (z.B. ein Grundschüler), können erfahrene Leser bereits am Wortbild selbst das jeweilige Wort erfassen, ohne einzelne Zeichen erkennen zu müssen (Hochuli 2005). Prägnantere Wortbilder, vergleiche hierzu linkes und rechtes Wort in Abbildung 1, könnten bei unerfahrenen Lesern den Prozess des Verinnerlichens unterstützen. Zur Einschätzung der Leseerfahrung könnte z.B. das Alter eines Lesers dienen oder die individuelle Auswertung der Augenbewegungen mittels Eye-Tracking, die in Echtzeit - innerhalb des Leseprozesses - die Form des Wortbildes je nach Beschaffenheit des Lesers beeinflusst (McConkie et al., 1991).
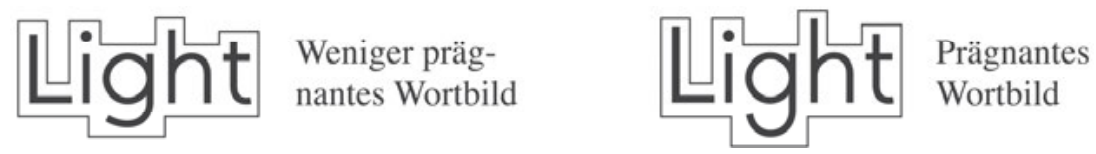

Abbildung 1: Die schematische Darstellung des Wortbildes. Links mit weniger, rechts mit einer hohen Prägnanz, da hier größere Über- und Unterlängen vorhanden sind. 


\subsection{Perspektive}

Durch die Auswertung des Standpunktes eines Betrachters relativ zum Ausgabemedium (zum Beispiel durch einen Kinect-Sensor), kann die perspektivische Verzerrung kompensiert werden. Das linke Bild in Abbildung 2 zeigt den optischen Eindruck des Betrachters, wenn dieser ein T von der Seite erblickt. Das rechte Bild, in Abbildung 2, zeigt die perspektivisch korrigierte Ansicht auf dem Ausgabegerät, um beim Betrachter den optischen korrekten Eindruck eines T's bei $90^{\circ}$ in Frontalperspektive zu gewährleisten. Dies kann insbesondere bei Verkehrsschildern oder Leitsystemen relevant sein.
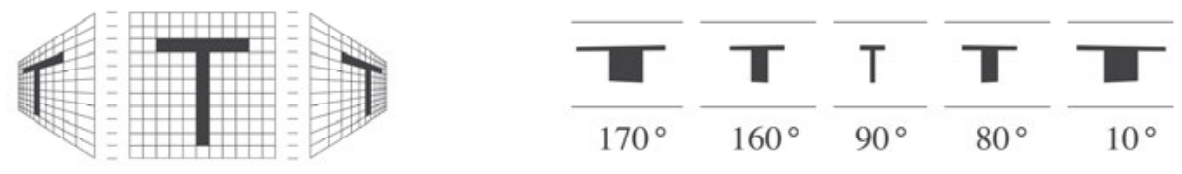

Abbildung 2: Die rechte Reihe an T's zeigt den Prozess der Anpassung an die Perspektive des Lesers.

\subsection{Fehlsichtigkeit}

Fehlsichtigkeit betrifft einen Großteil der Bevölkerung und wird auch durch Sehhilfen nicht immer optimal ausgeglichen. Kennt ein Ausgabegerät die Ametropie, sprich das Unvermögen eines Lesers scharf zu sehen, kann ein Zeichen so transformiert werden, dass dieses - unter der Unschärfe - eine höhere Prägnanz aufweist und somit besser von anderen Zeichen unterschieden werden kann. Die ungewöhnliche Zeichenform des rechten Y's, in Abbildung 3, kommt dabei nur für den Unscharf-Sehenden zum Einsatz - den Normalsehenden würde diese Zeichenform eher irritieren. Die Transformation des Zeichens bestimmt sich über die Dioptrien des Lesers. Die Fehlsichtigkeit wird entweder automatisch erkannt anhand seines Benutzerprofils oder der manuellen Justierung direkt am Lesemedium.

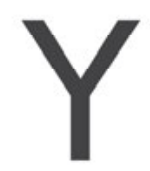

Original

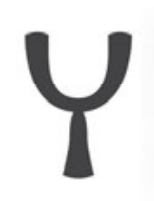

Korrigiert

Abbildung 3: Die Transformation des rechten Y's verbessert seine Erkennbarkeit unter der Unschärfe.

\section{$3 \quad$ Evaluierung}

Ein Schriftzeichen in seiner Zeichenform in Abhängigkeit zu den Eigenschaften seines Lesers zu stellen, eröffnet eine gänzlich neue Betrachtungsweise. Drei potentielle Möglichkeiten haben wir an ausgewählten Zeichen dargestellt. 
Bei der Evaluierung unseres Ansatzes kamen ganze Wörter und Wortfolgen zum Einsatz. Aufgrund der gegebenen Platzverhältnisse können wir nachfolgend nur auszugsweise auf die bisherigen Erkenntnisse eingehen, die anhand eines Fragebogens erhoben wurden. Mithilfe der Likert-Skala - trifft voll zu (1), trifft zu (2), teils-teils (3), trifft nicht zu (4), trifft überhaupt nicht zu (5) - wurde die Frage ,nach der generellen Akzeptanz reagierender Zeichen“ gestellt. Insgesamt wurde eine Punktezahl von 1.75 erreicht. Von den sieben Personen, zwei weiblich und fünf männlich im Alter zwischen 20 und 30 Jahren, die alle über sehr gute Kenntnisse in der Typografie verfügen, stand nur eine Person dem Thema neutral gegenüber, während alle anderen die Frage mit trifft voll zu bzw. mit trifft zu bewerteten.

Um die Frage der besseren Lesbarkeit zu beantworten, verwendeten wir sowohl objektive als auch subjektive Methoden. Anhand der Hausdorff-Metrik (Huttenlocher 1993) verglichen wir die Ähnlichkeit zwischen dem Originalzeichen und (a) dem Zeichen unter künstlicher Unschärfe sowie dem (b) optimal korrigierten Zeichen unter der gleichen künstlichen Unschärfe. Für mittlere Unschärfewerte war die Hausdorff-Metrik für (b) um ca. 10\% geringer als (a). Für große Unschärfewerte war (b) um ca. 7\% geringer als (a). Dies bedeutet, dass die reagierenden Zeichen besser in der Lage waren, die Form des Originalzeichens wiederzugeben. Doch spiegelt sich eine geringere Hausdorff-Metrik auch in einer besseren Lesbarkeit beim Menschen wieder? Hierfür fragten wir unsere sieben Probanden, welche Zeichen sie besser lesen können: Bei mittlerer Unschärfe bevorzugten $43 \%$ die reagierenden Zeichen (b). Mit ansteigender Unschärfe wurden die reagierenden Zeichen noch stärker bevorzugt bis schließlich $100 \%$ der Gefragten die reagierenden Zeichen, bei großer Unschärfe, bevorzugten.

\section{Literaturverzeichnis}

Huttenlocher, D. P., Klanderman, G. A., \& Rucklidge, W. J. (1993). Comparingimages using the hausdorff distance. In IEEE Transactions on Pattern Analysis and Machine Intelligence, vol. 15, no. 9, pp. 850-863.

Hochuli, J. (2005). Das Detail in der Typografie. Buchstabe, Buchstabenabstand, Wort, Wortabstand, Zeile, Zeilenabstand, Kolumne. Sulgen: Verlag Niggli AG, S. 8, S. 14

Kinross, R. (2010). Modern Typography. 2. Auflage. London: Hyphen Press.

Klanten, R., Mischler, M., Bilz, S., \& Thoenen, N. (2004). Type One. Berlin: Die Gestalten Verlag.

Marcottes, E. (2010). Responsive Webdesign In A List Apart. [Online]. http://alistapart.com/ article/responsive-web-design

McConkie, G.W., Zola, D., Grimes, J., Kerr, P.W., Bryant, N.R., \& Wolff P.M. (1991). Children's eye movements during reading. J.F. Stein (Ed.), Vision and visual dyslexia, CRC Press, S. 251-262

Smijers, F. (2011). Counterpunch, Making Type In The Sixteenth Century, Designing Typeface Now. 2. Auflage. London: Hyphen Press. 